\title{
IDENTIFICATION OF AIR TARGETS BY USING SECONDARY APPLICATION OF TERRESTRIAL TV
}

\author{
Yuri Kuznetsov*, Mikhail Cherniakov ${ }^{\infty}$, Andrey Baev* \\ * Moscow State Aviation Institute (Technical University) \\ $\infty$ The University of Birmingham, \\ Volokolamskoe shosse, 4, A-80, GSP-3, 125993, Russia \\ Tel.: 0070951584047 \\ Fax: 0070951581697 \\ E-mail: mai_k405@mtu-net.ru \\ Edgbaston B15 2TT, Birmingham, UK \\ Tel: 01214144286 \\ Fax: 01214144291 \\ E-mail: m.cherniakov@bham.ac.uk
}

\begin{abstract}
Numbers of bistatic radar systems based on non-cooperative TV transmitter are known currently. These systems provide air target detection and tracking but none of them have target identification ability. It is proposed in the paper a technique of air target identification based on the system transfer function parameters estimation. Modern terrestrial $T V$ stations in Europe are operating mainly in UHF band with channels spacing not less than 20MHz. Using, as an example, 4 channel transmitter operating at 480-540 MHz, the singularity expansion method is introduced. The technique based on the signatures of resonance models is suggested. These signatures are robust to range and aspect angle of targets and the method could be applied in practice.
\end{abstract}

\section{INTRODUCTION}

According to the Secondary Application of Wireless Technology (SAWT) concept [1] one of the challenge is an air target detection, tracking and identification by using radar based on non-cooperative TV transmitters. A simplified block-diagram of such a system is shown in Fig. 1.
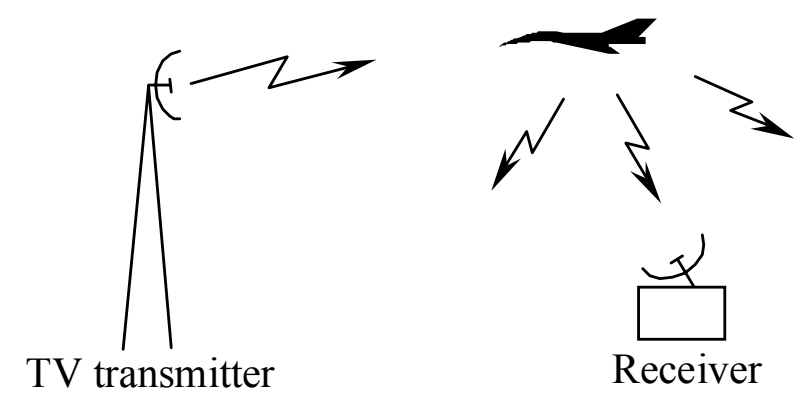

Fig. 1. Bistatic radar based on terrestrial TV

When targets detection and tracking in similar system is, in some instance, a solved problem, targets identification is a new and challenging task. So, the goal of the discussing research is to identify an air target using TV emitted signal as a ranging. Let's suppose that number of targets as aircrafts, missiles, etc., are simultaneously irradiated by electromagnetic field of four (or six in a future European standard) TV transmitters positioned at the same antennas tower. The transmitted signals are quadrature amplitude modulated (QAM) oscillations with central frequencies, say 480, 500, 520 and $540 \mathrm{MHz}$ and with $8 \mathrm{MHz}$ bandwidth each.

It is proposed targets identification via detection and parameters estimation of the natural resonances excited by multi channels TV signals. Advanced radar target discrimination methods have utilized the ultra-wideband exciting signals for these purposes. For example, in active radar stations are used the pulses with effective duration about nanoseconds without carrier frequency [2]. Unfortunately this method has very restricted area of applications, such as ground penetration radars due to electromagnetic compatibility problems. In the considered case exciting signals are located in a set of separated and relatively narrow frequency bands. Nevertheless this signal could be still specified as an ultrawideband. Consequently at least two problems are arising, namely: radar targets' natural frequencies parameters estimation and the second is to evaluate an influence of TV channels frequency allocations and bandwidth on the quality of identification process. Thus, it is required to identify physical objects (targets) by processing of four TV channels signals, i.e. to define the type of the airplane. The estimation of identification and discrimination quality for radar targets may be carried out by using natural frequencies (poles) and introduced in the paper signatures, which are invariant to the range and aspect angle of the targets. 


\section{RESONANCE MODELS}

According to the singularity expansion method (SEM) [3] it is possible to describe the physical object's scattering by its system transfer function

$$
H(s)=\frac{a_{0}+a_{1} s+a_{2} s^{2}+\ldots+a_{M} s^{M}}{1+b_{1} s+b_{2} s^{2}+\ldots+b_{N} s^{N}}=A \sum_{i=1}^{M}\left(s-s_{z i}\right) / \sum_{j=1}^{N}\left(s-s_{p j}\right)
$$

where $s_{z}$ are zeros and $s_{p j}=\sigma_{j}+i \omega_{j}$ are poles of the $H(s) ; \sigma_{j}, \omega_{j}$ are the $j$-th damping factor and natural frequency of the pole; $N$ is the number of dominant natural frequencies (resonances); $A$ is the constant.

If identifying objects have different shapes and/or made from different materials they have different system transfer functions. According to SEM the $H(s)$ is completely characterized by a set of natural frequencies in a complex $s$-plane. They are practically aspect independent and determined by size and shape of the object, when zeros are determined by an aspect angle and by the range to the object.

In Figure 2 are shown three general scattering modes in terms of the characteristic dimension of a target vs. wavelength of the ranging signals [4]. The natural frequencies of physical objects allocated in resonance area of frequencies and correspond to the characteristic target size. Therefore in an ideal case the frequency band of the exciting signal should overlap all resonance area of the object. For example, the object with characteristic sizes from 1 up to $10 \mathrm{~m}$ has resonance frequencies from $3 \mathrm{MHz}$ up to $3 \mathrm{GHz}$. TV signals of selected frequency band can excite resonance scattering for object with characteristic sizes from 0.03 up to 5 meters.

For example, it was considered two sets of the resonance poles on a complex $s$-plane, which correspond to the resonances of aircrafts [4]. Complex $s$-plane fourth quadrant pole plot of these aircraft models is depicted in Fig. 3. In this figure $p_{j}=\left(\sigma_{j}+i \omega_{j}\right) L / c \pi$, where $L$ is a characteristic size of the object, $c=3 \cdot 10^{8} \mathrm{~m} / \mathrm{s}$ is the velocity of light. Two frequency characteristics for the models of aircrafts are shown in Fig.4. The difference between spectrums of these models is big enough to separate and identify mentioned above models.

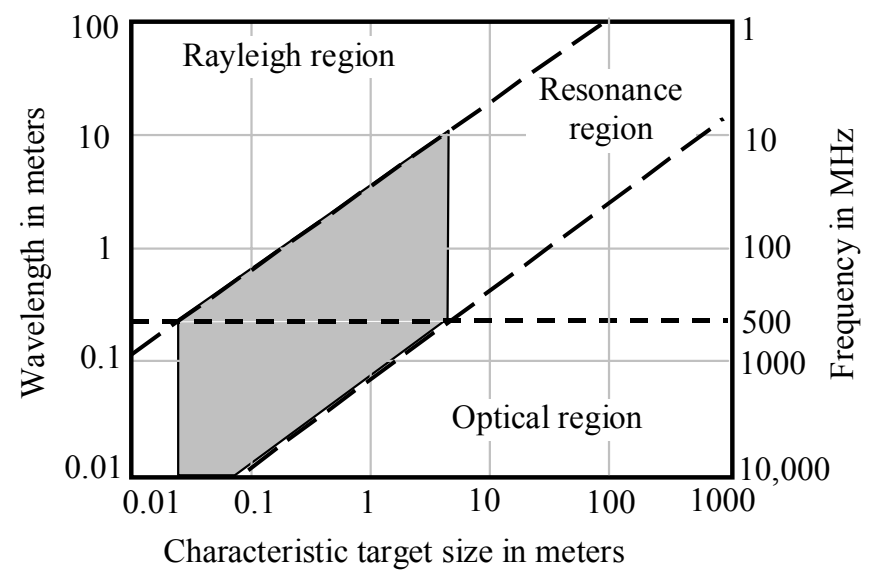

Fig. 2. Relationship of the three general scattering regions

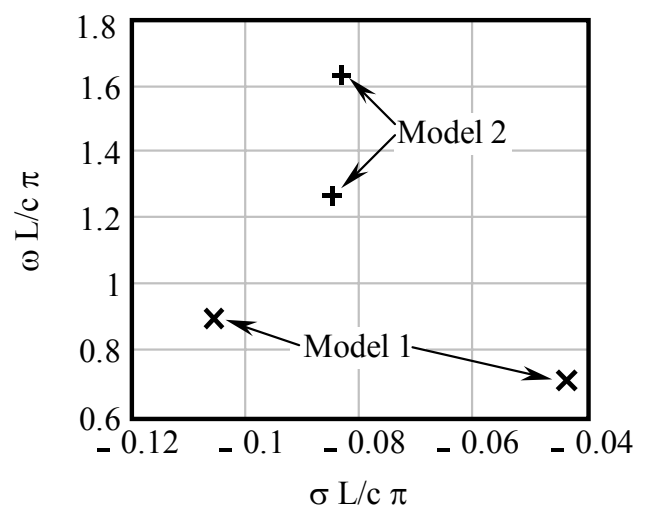

Fig. 3. Complex $s$-plane plot of aircraft models

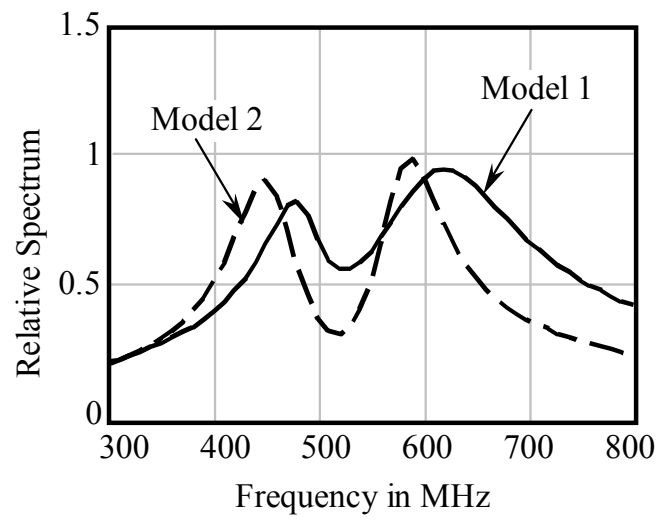

Fig. 4. Frequency characteristics of aircraft models 
The estimation of the system transfer function's poles may form the basis for targets identification. Frequency bands of the discussing radars are in the resonance area of these two models. However, their identification is affected by following problems: the spectrum of the TV signals is not completely overlaps the resonance area of the objects and the signal spectrum consists on four separated sub bands.

\section{IDENTIFICATION OF AIR TARGETS}

It is possible different ways of physical objects' identification by using measurements in frequency or time domains. The target response in the ranges of exciting signals is defined by the frequency characteristic, which may be calculated from the system transfer function $H(s)$ by replacement $s$ on $\{j 2 \pi f\}$ in (1). The amplitude-frequency characteristic's measurement can be carried out by matching amplitudes of irradiated TV signals and received signals in accordance to the range.

In our example we have taken the measured amplitude frequency responses $D_{k}, k=1, \ldots, K$ on $K=24$ frequencies located within the TV signals' frequency band with $1 \mathrm{MHz}$ step, which are shown in Fig. 5 by daggers. The frequency characteristic is depicted for the model 1. For the model 2 the measurement of the frequency characteristics was carried out similarly.

A measured amplitude frequency response was exposed to the digital processing to determine poles of system transfer function. Transfer functions presentations as the ratio of two complex polynomials from discrete frequency response data is, in essence, a rational approximation problem. Since the direct approach to the solution evaluation will result in non-linear equations, various alternative strategies have been suggested to solve the problem.

The least-squares approach has been used extensively in solving of various engineering problems. In our case the transfer function should be determined from the frequency response data. Parameters of the transfer function are to be selected to minimize the sum of the squared errors between the given response data $D_{k}$ and the approximating function $H\left(j 2 \pi f_{k}\right)$

$$
E=\sum_{k=1}^{K}\left(D_{k}-\left|H\left(j 2 \pi f_{k}\right)\right|\right)^{2} .
$$

The least-squares criterion always leads to non-linear equations relevant to unknown parameters. To avoid this an iterative procedure was applied to determine the transfer function from amplitude frequency response data alone.

Estimations of system transfer function's poles can be utilized for generation of targets signatures, which may form the basis for the machine processing of measured data. Each axis of signature space corresponds to the normalized poles' parameter of the resonance model. The reference points, which appropriate to parameters of identified objects, are located symmetrically relatively to the coordinate origins.

\section{RESULTS OF COMPUTER SIMULATIONS}

Utilization of the described above algorithm allows to obtain the frequency characteristics, shown in Fig. 6. For the given example an error in the poles' determination is not more, then units of percent, so the poles' estimations are practically similar to the true poles location.

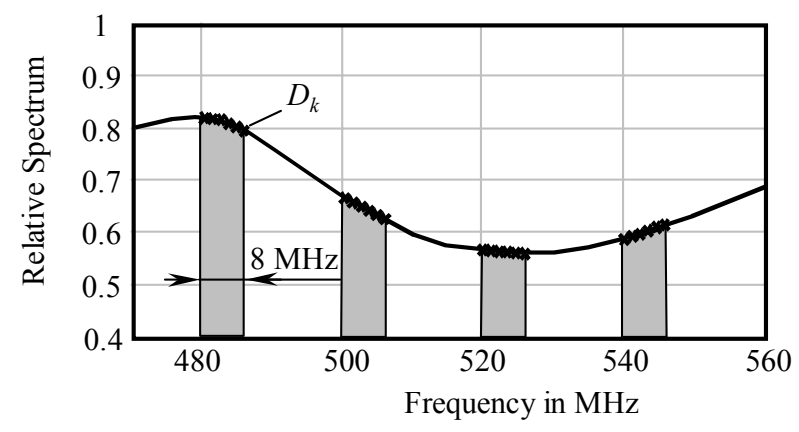

Fig. 5. Frequency characteristic for the model 1, where " $x$ " are the discrete values $D_{k}$ in the researched band

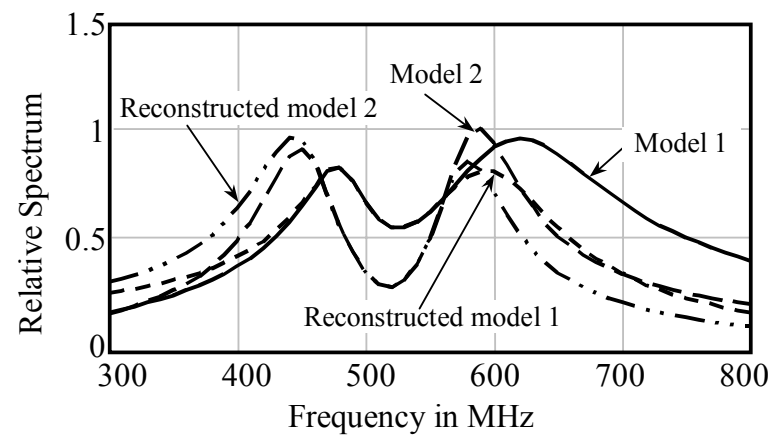

Fig. 6. Reconstructed frequency characteristics. 
We have simulated an influence of an aspect angle on an accuracy of the resonance model poles' estimation. According to the SEM an aspect angle variations could be executed by changing of system transfer function residues

$$
H(s)=\sum_{j=1}^{N} \frac{m_{j} b_{j}}{s-s_{p j}},
$$

where $b_{j}=\left|b_{j}\right| \mathrm{e}^{\arg \left(b_{j}\right)}$ is the residue of $j$-th pole $s_{j}$ and $m_{j}$ is the scale factor. Fig. 7 shows the estimations of poles for 50 different values of scale factors $m_{i}$, which were randomly changed from 0 to 10 . The positions of poles $s_{i}$ were accepted to be unchanged. Daggers depict the true positions of the resonance models' poles. From Fig. 7 it is seen that aspect's change lead to damping factor $\sigma_{j}$ variations, where frequencies $\omega_{j}$ of poles stay practically unchanged.

The change of the objects' characteristic sizes leads to the positioning of identified objects' poles outside frequency band covered by TV transmitters. It is accompanied by abrupt deterioration of resonance poles' estimation accuracy and by increase of distance between the measured and reference points in signature space (see Fig. 8).

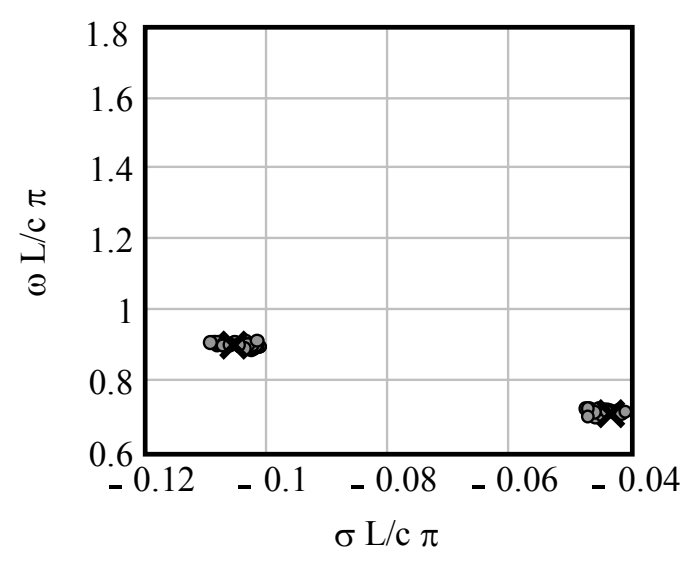

Fig. 7. Estimates of poles for 50 independent runs for the model 1

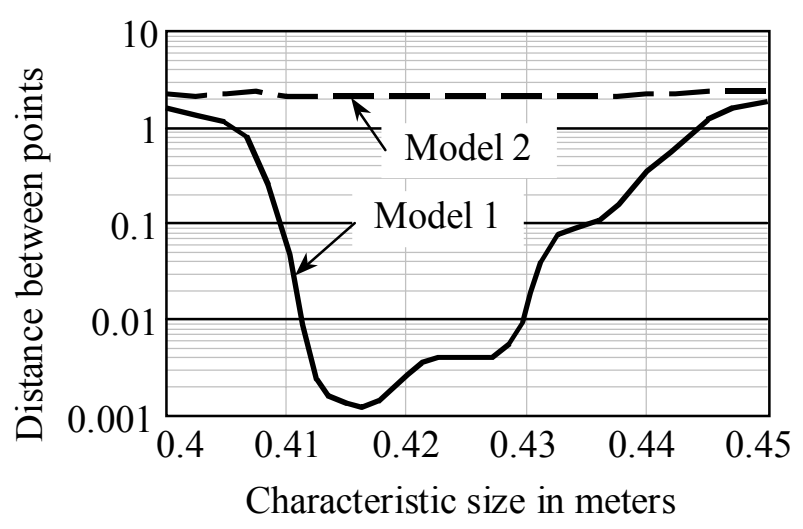

Fig. 8. Dependence of distance between measured and basic points in signature space

It is possible to state, that the poles of model practically are not determined, but objects' signatures have allowed expanding the range of identified objects' characteristic sizes, at which the right discrimination of objects is provided, in comparison with classical methods [3].

\section{CONCLUSIONS}

The presented results show target identification ability of the system, based on terrestrial TV in ultra-high frequency band. The identification may be realized by using the parameters estimation of system transfer function at measurement of amplitude-frequency characteristic in relatively narrow frequency band at 480 to $540 \mathrm{MHz}$. The boundaries of possible change of the characteristic sizes by air targets are determined. Inside these boundaries it is possible to have high probability of right identification by using signatures. It is shown that used signatures are practically invariant to range and aspect angle of targets.

\section{REFERENCES}

[1] Mikhail Cherniakov, Kurt Kubik, "Secondary Applications of Wireless Technology (SAWT): The Concept," in 2000 European Conference on Wireless Technology, Oct. 2000.

[2] Yury Kuznetsov, Andrey Baev, Rudolf Sedletskiy, “Application of E-pulse Method for Remote Sensing Arbitrary Shaped Objects in Lossy Media," in 30 th European Microwave Conference, pp. 255-259, Oct. 2000.

[3] C.E. Baum, "The Singularity Expansion Method," in Transient Electromagnetic Fields, L.B. Felson, Ed. New York: Springer-Verlag, 1976.

[4] Introduction to Ultra-Wideband Radar Systems, CRC Press, 1995. 